\title{
Temperature Characteristics and Error Compensation for Quartz Flexible Accelerometer
}

\author{
Jing-Min Gao ${ }^{1} \quad$ Ke-Bei Zhang ${ }^{1,2} \quad$ Fu-Bin Chen ${ }^{3} \quad$ Hong-Bo Yang ${ }^{3}$ \\ ${ }^{1}$ Electronic Information and Control Center, Beijing Information Science \& Technology University, Beijing 100085, China \\ ${ }^{2}$ Beijing Institute of Control Engineering, Beijing 100080, China \\ ${ }^{3}$ School of Automation, Beijing Information Science \& Technology University, Beijing 100085, China
}

\begin{abstract}
Reduction of error due to the influence of temperature on the quartz flexible accelerometer without any heating device is a difficult task, and is also a tendency for research and application. In this paper, static and dynamic temperature compensation models are established in order to reduce the temperature influence on accelerometer measurement accuracy. Combined with the experiment data, the relationship between the accelerometer output accuracy, temperature and the magnitude of acceleration is analyzed. The data collected from the temperature experiment show that output value of the accelerometer varies with temperature. The method of uniaxial quadrature experiment is adopted and the accelerometer output value is gauged at temperature ranging from $-20^{\circ} \mathrm{C}$ to $50^{\circ} \mathrm{C}$. Having used the static and the dynamic temperature compensation models, the accelerometer temperature error compensation experiment is conducted and the compensated errors by the two models are analyzed. The result shows that the compensated value meets the technical requirements. Two technical indicators, the zero bias $K_{0}$ and the scaling factor $K_{1}$, which are used to measure the degree of accelerometers, are both improved and their fluctuation ranges are reduced.
\end{abstract}

Keywords: Acceleration, error compensation, temperature characteristics, quadrature experiment, differential mode.

\section{Introduction}

Quartz flexible accelerometer is one of the core components of the inertial navigation and inertial guidance system. The temperature coefficients of accelerometer core and the core torque, which jointly determine the accelerometer output accuracy, are both affected by the temperature. In practical applications, it is essential to take measures to reduce the impact of temperature on the accelerometer output value. Two coefficients related to the accelerometer are introduced to measure the effect of the various temperatures posed on the accelerometer. The two coefficients are referred to as the scaling factor $K_{1}$ and the zero bias $K_{0}$. The slope of the curve, namely $K_{1}$, representing function relation between output signal and measured acceleration, serves as a major characteristic of accelerometer. The zero bias output single, namely $K_{0}$, reflects the magnitude of accelerometer output value when the input acceleration is zero $^{[1-3]}$. Due to the fact that the characteristics of circuit used to process output signal in accelerometer is determined by temperature, the values of two indicators are different for different temperature to another. Studies prove that the more is robustness of $K_{1}$ against temperature, the higher is the quality level of accelerometer. For some specific appli-

\footnotetext{
Regular Paper

Manuscript received September 12, 2013; accepted November 18, 2014

Recommended by Associate Editor Xun Chen

This work was supported by the Importation and Development of High-caliber Talents Project of Beijing Municipal Institutions (No. IT\&TCD201304115).

(C) Institute of Automation, Chinese Academy of Science and Springer-Verlag Berlin Heidelberg 2015
}

cation such as spacecraft or satellite, the temperature instability $K_{1}$ should not be exceed $0.1 \%$ in complete range of operating temperature ${ }^{[4,5]}$. However, the spacecraft is moving so fast that the external ambient temperature changes dramatically. If the ambient temperature difference is up to $10^{\circ} \mathrm{C}$ or even more, the error of output value will be ranging from $0.12 \mathrm{mG}$ to $1.405 \mathrm{mG}$ (the value can be illustrated by the Figs. 4 and 5). The symbol $\mathrm{G}$ is short for gravity unit. The unit $\mathrm{G}$ is equal to $1000 \mathrm{mG}$, and also equal to $10^{6} \mathrm{uG}$. The speed and position error can be estimated as

$$
\left\{\begin{array}{l}
\Delta V=\int_{0}^{t} \alpha(t) \mathrm{d} t \\
\Delta S=\iint_{0}^{t} \alpha(t) \mathrm{d} t
\end{array}\right.
$$

where $\alpha(t)$ is the acceleration error caused by the temperature. The variable $\Delta V$ and $\Delta S$ are the errors of the speed and position, respectively. The variable $t$ reflects how long the temperature is changing dramatically. If the variable $t$ is reaching to tens of minutes, the errors $\Delta V$ and $\Delta S$ are so large that it is a disaster for the spacecraft or satellite. Therefore, it is necessary to study the temperature characteristics of accelerometer and compensate the output value.

In practical application, there are two ways to reduce the influence of temperature on the quartz flexible accelerometer. The one is to add a temperature control device on accelerometer. For example, Yu et al. ${ }^{[6,7]}$ used the first method. They designed a two-stage robust temperature control device. It was observed that there was almost no change in the accelerometer temperature. But both the big size of the device and the power consumed by the device are not feasible in the aerospace field. Eichstat et al. ${ }^{[8,9]}$ 
tried to use software and hardware method to do accelerometer temperature compensation, but they did not give any high precision algorithm. Shinamiya ${ }^{[10]}$ proposed two-phase induction method to analyze the temperature error and conduct the temperature compensation. Shinamiya inserted the temperature compensation circuit to the output winding of the accelerometer. The second way to reduce the influence of temperature is to study the temperature compensation algorithm. The neural network algorithm is used to compensate the accelerometer output in references ${ }^{[5,11-15]}$. The accelerometer precision is well proved by the neural network algorithm. But the complexity of neural network makes it inappropriate to do the temperature compensation of accelerometer used in aerospace field. The relevant vector machine algorithm was used to compensate thermal bias drift in [16] and a model which contained the temperature and the temperature rate was established. The experimental result proved that thermal bias drift of the accelerometer can be fitted accurately by the model and the mean square error is less than $1 \%$. The static temperature compensation algorithm was given in [17] and temperature drift could be reduced properly. The dynamic temperature compensation algorithm was proposed in [18]. But the rate of change of temperature was not considered in the dynamic compensation. In a word, an accelerometer temperature compensation model, which considers the temperature and the temperature rate, is helpful to improve the precision of accelerometer. It is also the focus of this paper. The paper proposes a static temperature compensation model and a dynamic temperature compensation model. The temperature and the temperature rate are considered in the dynamic model.

The organization of the paper is as follows. Descriptions of the experiment steps are provided in Section 2. Section 3 describes the static temperature model and the results of compensation are shown in Section 4 . The dynamic temperature models and results of compensation are described in Section 5. Finally, some conclusions are given in Section 6 .

\section{Experiment step}

The experimental setups and implementation steps should be first considered before the conduct of temperature experiment. The experimental installation diagram is shown in Fig. 1. The whole experiment setup is consisted of 5 sections, the power supply and computer. As shown in Section 1, the rotating disc, which is used to alter the accelerometer input, can be turned in clockwise and counter-clockwise direction. The accuracy of rotating disc is less than 5. The length of rotating disc axis is not greater than $20 \mathrm{~cm}$. The diameter of the rotating disc axis is no more than $10 \mathrm{~cm}$. Therefore, in the vertical direction, the accelerometer centroid fluctuation ranges from 0 to $20 \mathrm{~cm}$. The impact of the gravitational field imposed on the accelerometer is less than $0.0314 \mu \mathrm{G}$. The value is so negligible that it can be ignored. Section 2 describes a thermostat, which is used to change the ambient temperature of accelerometer. The accuracy of the thermostat is less than $0.5^{\circ} \mathrm{C}$. Two accelerometers, which are the main objects for the temperature experiment, are installed in Section 3. The relationship between accelerometer output-input values and the temperature is the prime target to research. Near the accelerometer, a temperature sensor named DS18B20 is fixed, shown in Section 4. The sensor is designed to test the temperature of components around accelerometer. Section 5 is designed to collect the output value of temperature sensor and accelerometer. The frequency of collection circuit is greater than $10 \mathrm{~Hz}$. When the high-precision algorithmic compensation of temperature is finished, the coefficients are downloaded to the compensation module of circuit. Then, accelerometer output value can be compensated in time.

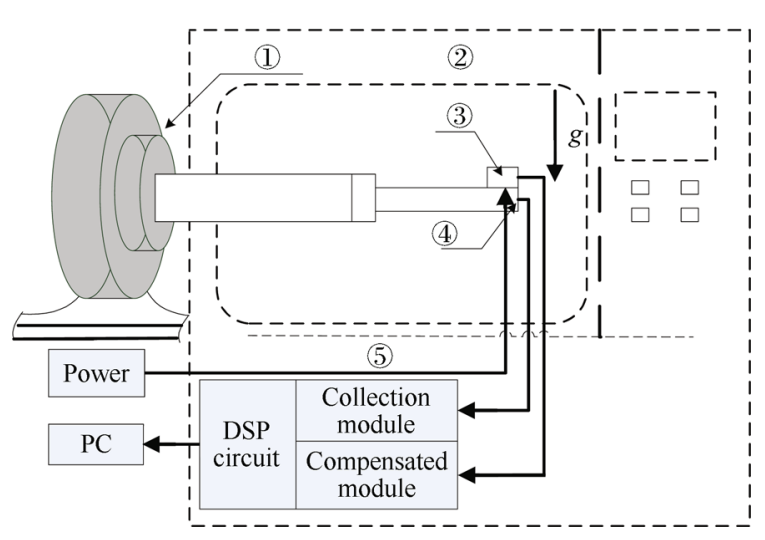

Fig. 1 Schematic diagram for experimental setup

The experiments used to analyze temperature characteristics of quartz accelerometers can be divided into 2 categories. One is gravity field flip experiment and the other is centrifuge experiment. The 4 points and 8 points, methods are popularly used in gravity field flip experiment. However, the 12 points biaxial orthogonal method is adopted in the paper. Accelerometers are installed on the rotating disc as shown in Fig. 2. Accelerometers A and B are mounted on precision hexahedron. The angle between accelerometers $\mathrm{A}$ and $\mathrm{B}$ is $90^{\circ}$. When the rotating disc is turned one circle in clockwise or counter-clockwise direction, the accelerometer input ranges from $-1 \mathrm{G}$ to $1 \mathrm{G}$. Then, the accelerometers output values are written down. The 12 points include $0^{\circ}$, $30^{\circ}, 60^{\circ}, 90^{\circ}, 120^{\circ}, 150^{\circ}, 180^{\circ}, 210^{\circ}, 240^{\circ}, 270^{\circ}, 300^{\circ}$ and $330^{\circ}$. For example, when the accelerometer $\mathrm{A}$ is at the point $0^{\circ}$, the accelerometer $\mathrm{B}$ is at the point $90^{\circ}$.

The output values at every point $\left(0^{\circ}, 30^{\circ}, 60^{\circ}, 90^{\circ}, 120^{\circ}\right.$, $150^{\circ}, 180^{\circ}, 210^{\circ}, 240^{\circ}, 270^{\circ}, 300^{\circ}$ and $\left.330^{\circ}\right)$ are collected by a 24 -bit $\mathrm{A} / \mathrm{D}$ circuit. In clockwise direction, a set of data at each point is acquired as $E_{1 j}(j=1,2, \cdots, 12)$. In counterclockwise direction, another set of data at each point is acquired as $E_{2 j}$. The average value at every point can be calculated as (2).

$$
E_{j}=\frac{E_{1 j}+E_{2 j}}{2}
$$




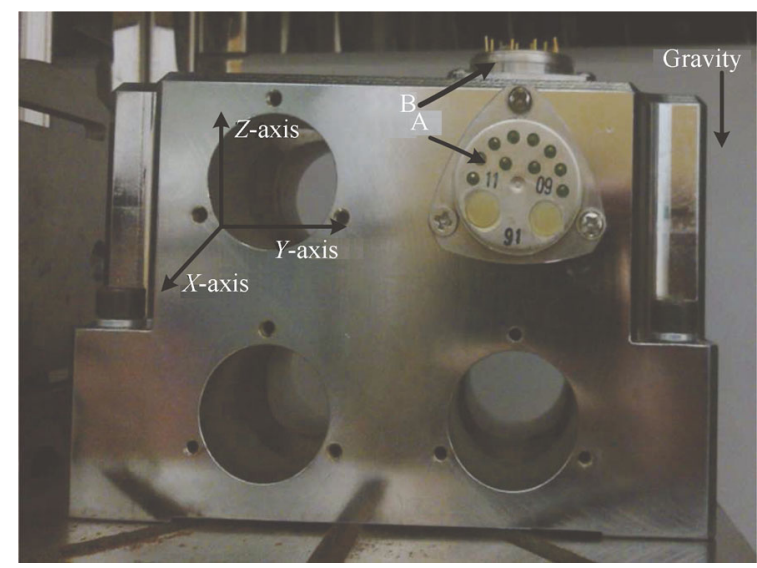

Fig. 2 Installation axis of accelerometer

As shown in Fig. 2, the measurement coordinate system includes 3 axes. They are $X$-axis, $Y$-axis and $Z$-axis. Accelerometer $\mathrm{A}$ is installed on the $X$-axis and accelerometer $\mathrm{B}$ is installed on the $Z$-axis. The rotating disc is rotated about $Y$-axis. The direction of gravity is perpendicular to the ground or $Y$-axis. When the rotating disc is rotated, the $X$-axis and $Z$-axis are turned vertically. The coordinate system of pendulum in accelerometer is inconsistent with the measurement coordinate system. In temperature experiment, the measurement coordinate system for every accelerometer is different. Difference between measurement coordinate system and pendulum coordinate system is dependent on the different installation tool. But the pendulum coordinate system is the same for every accelerometer, which is dependent on the accelerometer craftsmanship. Three axes are considered in the coordinate system of pendulum. For a 3 axes accelerometer, it is required to consider the difference between the 2 coordinate systems. Therefore, the mounting error angles $\theta_{i}$ and $\theta_{p}$ exist. Installation error angles $\theta_{i}$ and $\theta_{p}$ are both small angles. The error angles $\theta_{i}$ and $\theta_{p}$ are determined by the hexahedral accuracy and installation accuracy. The method to reduce the effect of error angle imposed on the accelerometer output is based on the creation of a bi-axial orthogonal model ${ }^{[19,20]}$. For understanding the relationship between temperature and the accelerometer's output value, the experiments under various temperature points are done. The experimental steps are important to note, which are as follows:

1) To install the accelerometers. The accelerometer mechanical zero position is often inconsistent with the electrical output zero position. Rotate the dividing degree head $180^{\circ}$ several times in clockwise and counterclockwise in order to find the point of the minimum output value. Then, the point is marked as the accelerometer mechanical zero position.

2) The accelerometers should be preheated 1 hour. Then, the experiment can be performed.

3) To identify the accelerometer temperature model and the simplified data processing, a method called 12 points is implemented. These 12 dividing points are $0^{\circ}, 30^{\circ}, 60^{\circ}$, $90^{\circ}, 120^{\circ}, 150^{\circ}, 180^{\circ}, 210^{\circ}, 240^{\circ}, 270^{\circ}, 300^{\circ}$ and $330^{\circ}$.
4) To meet the requirements of temperature range in practical application, the temperature in the experiment ranges from $-20^{\circ} \mathrm{C}$ to $50^{\circ} \mathrm{C}$. The method named 12 points was used at intervals of $10^{\circ} \mathrm{C}$ temperature points in experiments.

\section{Static compensation model}

To find out the actual state of the accelerometer, 3 axes are considered to discuss the input-output relationship of the accelerometer. They are the input reference axis (IA), the pendulum reference axis (PA) and the output reference axis (OA). The orientations of the 3 axes are determined by the right-hand rule ${ }^{[21,22]}$. The relationship between the 3 axes is shown in Fig. 3. Under the action of gravity, the accelerometer output value of 3 axes can be illustrated as (3).

$$
\left\{\begin{array}{l}
\alpha_{i}=\sin (\theta) \\
\alpha_{p}=-\cos (\theta) \\
\alpha_{o}=0
\end{array}\right.
$$

where the variable $\theta$ is the rotational angle, which is the angle between the accelerometer $\mathrm{A}$ and the horizontal line.

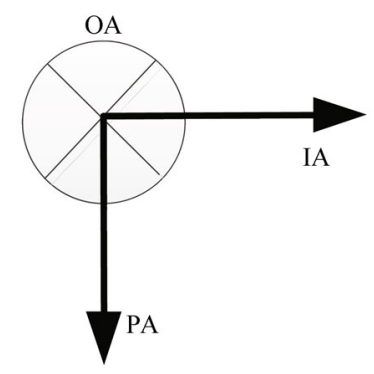

Fig. 3 The reference axis of accelerometer

The accelerometer model equation is the mathematical relationship between output value $E$ and acceleration's value along the 3 reference axes. The mathematical relationship is shown as

$$
E=K_{1}\left(K_{0}+\alpha_{i}+K_{2} \alpha_{i}^{2}+K_{i p} \alpha_{i} \alpha_{p}+K_{i o} \alpha_{i} \alpha_{o}\right)
$$

where the variable $E$ is the output value of accelerometer. $K_{0}$ represents the zero bias $(G), K_{1}$ represents the thermometric scale factor $(V / G), K_{2}$ represents the second-order nonlinear coefficient $\left(G / G^{2}\right)$. The variable $\alpha_{i}$ represents the accelerometer's value along the IA-axis $(G)$. The variable $\alpha_{p}$ represents the accelerometer's value along the PA-axis $(G)$. The variable $\alpha_{o}$ represents the accelerometer's value along the OA-axis $(G)$. The angle $\theta$ in (3) is changed by the rotating disc. Known from (3), the input values of $\alpha_{i}$ and $\alpha_{p}$ can be calculated out. For example, when the angle $\theta$ is 0 , the value of $\alpha_{i}$ is 0 and the value of $\alpha_{p}$ is $-1 \mathrm{G}$. When the angle $\theta$ is $90^{\circ}$, the value of $\alpha_{i}$ is $1 \mathrm{G}$ and the value of $\alpha_{p}$ is 0 . The characteristic of output value varies with temperature and input acceleration. The relationship between the output value and temperature is established 
by repeated experimental tests performed in several days. The accelerometer A output values at point $90^{\circ}$ are shown in Fig. 4 and the accelerometer B output values at point $270^{\circ}$ are shown in Fig. 5. Data in Figs. 4 and 5 indicate that output value is decreasing when it is greater than 0 . When the output value is not greater than 0 , the absolute value of the output is also decreasing. Given certain acceleration, the accelerometer measurement values fluctuated considerably at different temperatures, resulting in degradation of accelerometer measurement accuracy. Moreover, several measurements show that the law under which the accelerometer output value varied with temperature can be given by (5). Equation (5) reflects a second order function, i.e., the output voltage is related with temperature and acceleration. In (5), the variable $E$ represents the output value of the accelerometer. The variable $t$ represents the temperature $\left({ }^{\circ} \mathrm{C}\right)$. The variable $\alpha$ represents input acceleration.

$$
E=f(t, \alpha)
$$

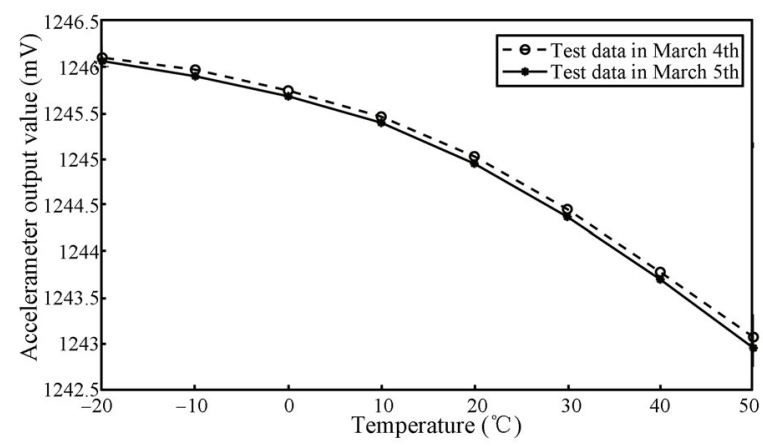

Fig. 4 Accelerometer A output value at $90^{\circ}$

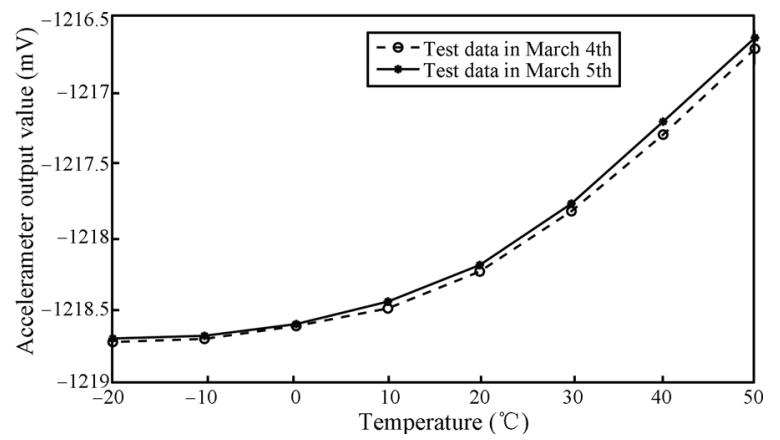

Fig. 5 Accelerometer B output value at $270^{\circ}$

As shown in Figs. 4 and 5, the output value curves of accelerometer are the result of repeated tests performed in several days. By changing the external ambient temperature, the output value of accelerometer fluctuates dramatically. For example, when the external ambient temperature is changing by $10^{\circ} \mathrm{C}$, the variation difference of accelerometer output value is up to $0.5 \mathrm{mV}$. The variation difference of accelerometer output value is up to $2.25 \mathrm{mV}$ or even more, when the ambient temperature ranges from $50^{\circ} \mathrm{C}$ to $-20^{\circ} \mathrm{C}$. In other words, when the input acceleration is given, the error of output value will be fluctuating from
$0.12 \mathrm{mg}$ to $1.405 \mathrm{mg}$ because of fluctuation the external ambient temperature caused ${ }^{[14-18]}$. The variation difference of accelerometer output value is reduced as the temperature is escalated.

The accelerometer output voltage at room temperature $20^{\circ} \mathrm{C}$ can be seen as a reference voltage. When the ambient temperature of the accelerometer is at other value, the temperature compensation for the output value is needed. The difference voltage between accelerometer output value at $20^{\circ} \mathrm{C}$ and other temperatures is called $\Delta E^{[23,24]}$. From (4), it can be known that the difference voltage $\Delta E$ is also related with temperature and acceleration. Therefore, the difference voltage $\Delta E$ can be calculated as

$$
\begin{aligned}
& \Delta E=f(t, \alpha)-f(20, \alpha)= \\
& \quad k_{00}+k_{01} \times t+k_{02} \times t^{2}+\left(k_{10}+k_{11} \times t+k_{12} \times t^{2}\right) \times \\
& \\
& \alpha+\left(k_{20}+k_{21} \times t+k_{22} \times t^{2}\right) \times \alpha^{2} .
\end{aligned}
$$

Experimental results obtained in several days certify that the difference voltage $\Delta E$ can be well described by (5). By using Matlab, the difference voltage $\Delta E$ can be fitted perfectly. Also, coefficients in (6) can be calculated easily. The difference voltage $\Delta E$ of accelerometer A at every temperature point is illustrated in Figs. 6-9. The curves in Figs. $6-9$ show that $\Delta E$ varies with temperature. From the temperature curve in Figs. 6-9, every curve reflects that $\Delta E$ is related with the value of acceleration at a static temperature point. The difference voltage $\Delta E$ changes linearly with the increase of the acceleration when the accelerometer is in the 1st or 2nd quadrant. The difference voltage $\Delta E$ increases when the temperature is greater than $20^{\circ} \mathrm{C}$ and the acceleration increases. The difference voltage $\Delta E$ decreases in other situation. When the accelerometer is in the 3rd or 4th quadrant, the trend of $\Delta E$ is similar with it as in the 1st or 2nd quadrant. From the horizontal axis in Figs. 6-9, it is observed that the difference voltage $\Delta E$ is related with the temperature. Given the input acceleration, $\Delta E$ value is the point on the curves or between them. Using the algorithm of least-squares, the $\Delta E$ value in each quadrant can be fitted into a two-order temperature model. Then, all the coefficients in (4) can be calculated. The coefficients of accelerometer A and B are shown in Tables 1 and 2 , respectively.

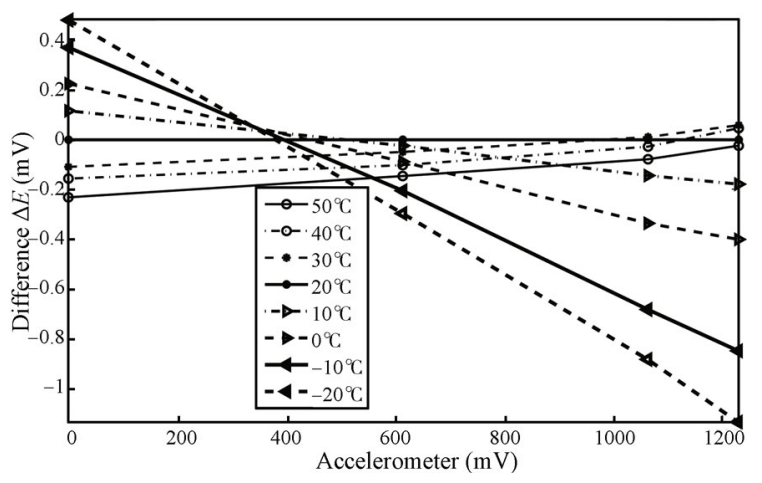

Fig. 6 The $\Delta E$ value in the 1st quadrant 


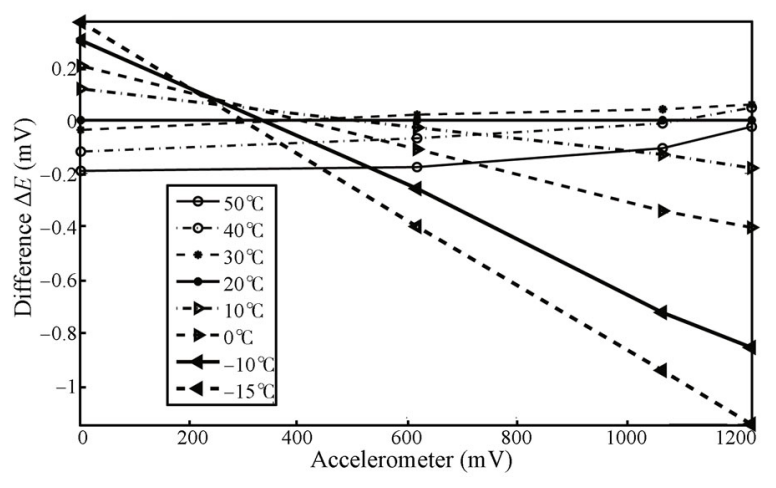

Fig. 7 The $\Delta E$ value in the 2 nd quadrant

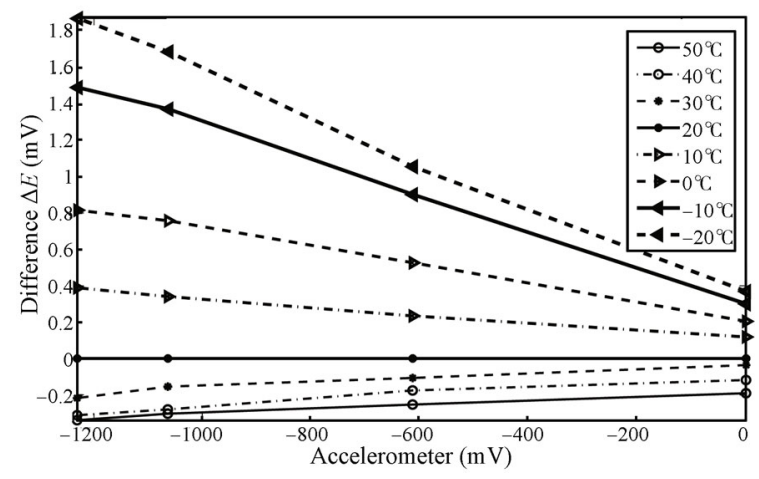

Fig. 8 The $\Delta E$ value in the 3rd quadrant

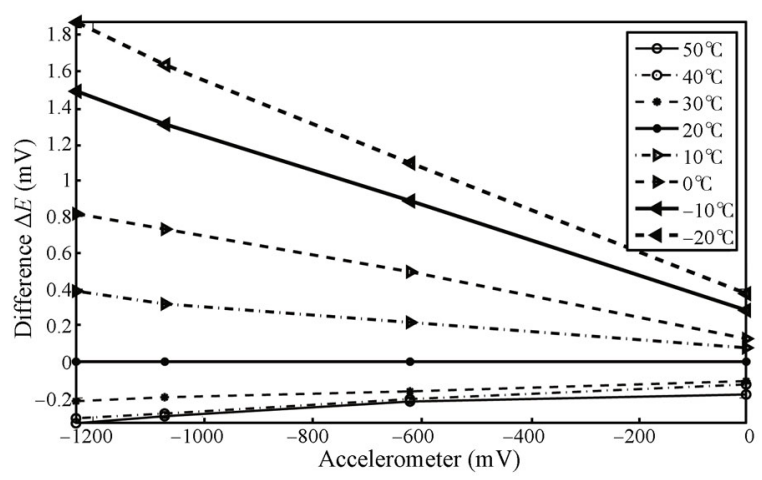

Fig. 9 The $\Delta E$ value in the 4 th quadrant

Table 1 The accelerometer A coefficients

\begin{tabular}{ccccc}
\hline Coefficient & $\begin{array}{c}\text { The 1st } \\
\text { quadrant }\end{array}$ & $\begin{array}{c}\text { The 2nd } \\
\text { quadrant }\end{array}$ & $\begin{array}{c}\text { The 3rd } \\
\text { quadrant }\end{array}$ & $\begin{array}{c}\text { The 4th } \\
\text { quadrant }\end{array}$ \\
\hline$K_{2,2}$ & -3 & 10 & 2 & 3 \\
$K_{2,1}$ & 287 & -269 & -258 & -520 \\
$K_{2,0}$ & -1770 & 1078 & 3410 & 9938 \\
$K_{1,2}$ & -46 & -59 & -47 & -42 \\
$K_{1,1}$ & 3602 & 3927 & 3463 & 2710 \\
$K_{1,0}$ & -55607 & -55547 & -50460 & -37783 \\
$K_{0,2}$ & 9 & 5 & 5 & 9 \\
$K_{0,1}$ & -1479 & -994 & -987 & -1489 \\
$K_{0,0}$ & 25035 & 19946 & 19814 & 25170 \\
\hline
\end{tabular}

Table 2 The accelerometer B coefficients

\begin{tabular}{ccccc}
\hline Coefficient & $\begin{array}{c}\text { The 1st } \\
\text { quadrant }\end{array}$ & $\begin{array}{c}\text { The 2nd } \\
\text { quadrant }\end{array}$ & $\begin{array}{c}\text { The 3rd } \\
\text { quadrant }\end{array}$ & $\begin{array}{c}\text { The 4th } \\
\text { quadrant }\end{array}$ \\
\hline$K_{2,2}$ & -1 & 4 & 9 & -8 \\
$K_{2,1}$ & -1622 & 1183 & 1064 & -1586 \\
$K_{2,0}$ & 18484 & -13282 & -12409 & 16363 \\
$K_{1,2}$ & -37 & -40 & -27 & -47 \\
$K_{1,1}$ & 210 & -1955 & -818 & -2725 \\
$K_{1,0}$ & -2110 & 24963 & 9879 & 32543 \\
$K_{0,2}$ & -11 & -12 & -12 & -11 \\
$K_{0,1}$ & -326 & -2140 & -2151 & -316 \\
$K_{0,0}$ & 4586 & 21642 & 21746 & 4455 \\
\hline
\end{tabular}

\section{Static compensation result}

Using the coefficients in Tables 1 and 2, the changes in difference voltage $\Delta E$ can be calculated no matter what the temperature and acceleration is. The compensated voltage is calculated as

$$
\begin{aligned}
\bar{E}= & E-\Delta E=E-\left\{k_{00}+k_{01} \times t+k_{02} \times t^{2}+\right. \\
& \left(k_{10}+k_{11} \times t+k_{12} \times t^{2}\right) \times \alpha+ \\
& \left.\left(k_{20}+k_{21} \times t+k_{22} \times t^{2}\right) \times \alpha^{2}\right\}= \\
& E-\Delta E=E-\left\{k_{00}+k_{01} \times t+k_{02} \times t^{2}+\right. \\
& \left(k_{10}+k_{11} \times t+k_{12} \times t^{2}\right) \times v+ \\
& \left.\left(k_{20}+k_{21} \times t+k_{22} \times t^{2}\right) \times v^{2}\right\}
\end{aligned}
$$

where the variable $\alpha$ is the input of accelerometer. The variable $v$ represents the accelerometer output voltage value when the input acceleration is $\alpha$. In the compensated section, one problem should be considered. That is which quadrant coefficients should be used to calculate the difference voltage $\Delta E$. To solve this problem, both the output voltages of accelerometers A and B are considered. The relationship between quadrant number and the output voltage of accelerometers A and B is shown in Table 3. From Table 3 , it can be seen that the quadrant numbers vary with the output voltage of the accelerometers. For example, if both the voltage $E_{1}$ and $E_{2}$ are greater than zero, then the 1st quadrant coefficients in Table 1 are adopted to calculate the difference voltage $\Delta E$ for accelerometer $\mathrm{A}$ and the 2nd quadrant coefficients in Table 2 are adopted to calculate the difference voltage $\Delta E$ for accelerometer B. Using (7) and the relationship shown in Table 3 , the difference voltage $\Delta E$ can be calculated no matter what point the accelerometer is in. Then, the compensated value $E$ can be calculated. Experiments show that the accuracy of output value after compensation is improved greatly. When the temperament ranges from $-20^{\circ} \mathrm{C}$ to $50^{\circ} \mathrm{C}$, The variation difference after compensation is no more than $0.05 \mathrm{mV}$ and the variation difference without compensated is up to $2.25 \mathrm{mV}$.

Two indicators are usually considered to evaluate the compensation method. In order to evaluate the effectiveness of the temperature compensation, the technical indicators before and after temperature compensation should be calculated ${ }^{[25-27]}$. Indicators $K_{0}$ and $K_{1}$ can be calcu- 
lated as

$$
\left\{\begin{array}{l}
K_{1}=A_{1}+3 A_{2} \\
K_{0}=A_{0}+B_{1}
\end{array}\right.
$$

Table 3 Relationship between quadrant number and output voltage

\begin{tabular}{clll}
\hline Output voltage & Output voltage & $\begin{array}{c}\text { A quadrant } \\
\text { number }\end{array}$ & $\begin{array}{c}\text { B quadrant } \\
\text { number }\end{array}$ \\
\hline$E_{1} \geq 0$ & $E_{2} \geq 0$ & 1 st & 2 nd \\
$E_{1} \geq 0$ & $E_{2}<0$ & 2 nd & $3 \mathrm{rd}$ \\
$E_{1}<0$ & $E_{2} \leq 0$ & $3 \mathrm{rd}$ & $4 \mathrm{th}$ \\
$E_{1}<0$ & $E_{2}>0$ & $4 \mathrm{th}$ & $1 \mathrm{st}$ \\
\hline
\end{tabular}

The coefficients $A_{0}, A_{1}, A_{2}$ and $B_{1}$ in (8) can be calculated as

$$
\left\{\begin{array}{l}
A_{0}=\frac{1}{n} \sum_{j=0}^{n-1} E_{j} \\
A_{1}=\frac{2}{n} \sum_{j=0}^{n-1} E_{j} \sin \alpha_{n} \\
A_{2}=\frac{2}{n} \sum_{j=0}^{n-1} E_{j} \sin 3 \alpha_{n} \\
B_{1}=\frac{2}{n} \sum_{j=0}^{n-1} E_{j} \cos \alpha_{n}
\end{array}\right.
$$

where the variable $E_{j}$ represents the average value at every point given by (2). The variable $n$ represents the point number. The variable $\alpha$ represents the angle the rotating disc turns. In the temperature compensation section, two set of data can be collected. The one is the accelerometer output value without compensation. The other is the accelerometer output value after compensation. Using the two set of data, two indicators $K_{0}$ and $K_{1}$ can be calculated using (9). Both the compensation results of accelerometers $\mathrm{A}$ and $\mathrm{B}$ are shown in Figs. 10-13.

\section{Dynamic compensation model}

The static temperature compensation value at different temperatures can be calculated by the static temperature model, which resolves the static temperature compensation issues. When the external ambient temperature is in the process of changing, the different value in internal temperature of the accelerometer is smaller than that of ambient temperature. The reason is that inertia transform is one of the essential properties of the temperature. The internal temperature lags behind external ambient temperature. The change in internal temperature is so small that the difference value of accelerometer output is smaller. If the static temperature model and measuring temperature were adopted to calculate the compensation value, the compensation effect would be worse. Therefore, the dynamic temperature conduction model is established essentially for accelerometer itself. The dynamic model transforms the external ambient temperature $T_{o}$ to the internal temperature
$T_{i}$ in a certain mathematical function when the external ambient temperature changes rapidly. Both the variable

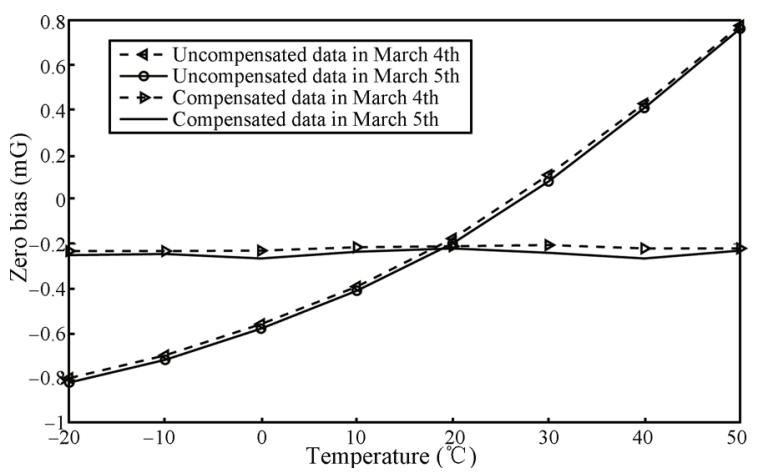

Fig. 10 Indicator $K_{0}$ for accelerometer A

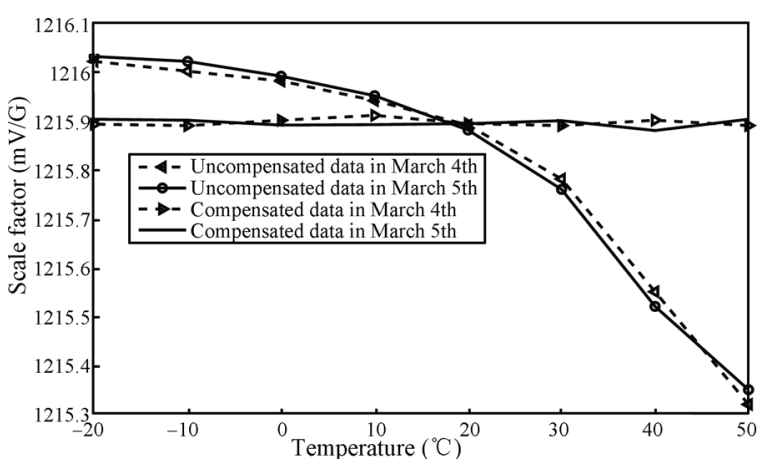

Fig. 11 Indicator $K_{1}$ for accelerometer A

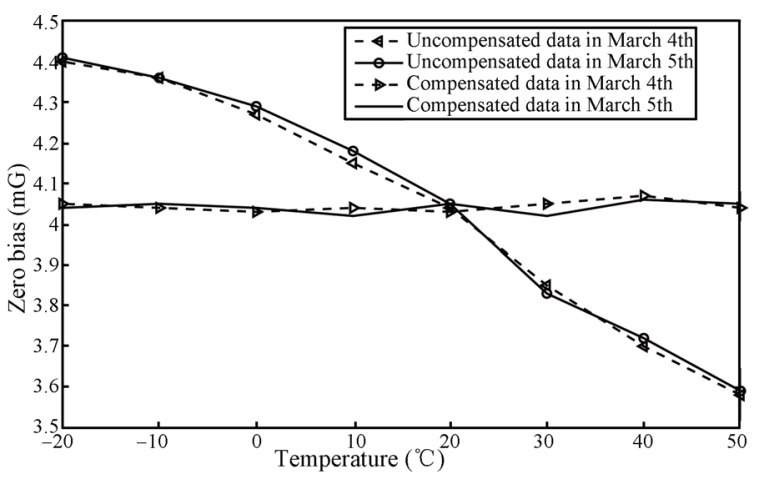

Fig. 12 Indicator $K_{0}$ for accelerometer B

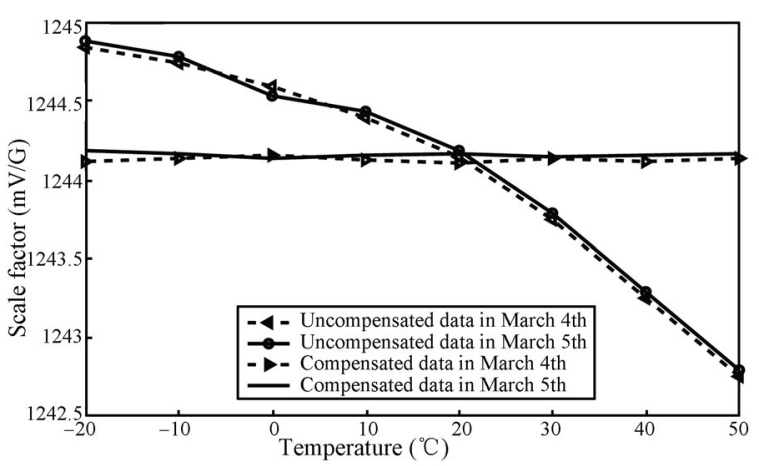

Fig. 13 Indicator $K_{1}$ for accelerometer B

$T_{i}$ and the static model are adopted to calculate the compensation value $\Delta E$. Simulation results illustrate that the 
dynamic model is effective and the accuracy of output value is improved. From the static model, it can be known that the output value is uniquely determined when the temperature reaches equilibrium. In other word, the static temperature. Therefore, the internal temperature can be calculated by the output value. The curves of external ambient temperature and internal temperature are shown in Fig. 14. Three curves, the step temperature curve, actual temperature curve and internal estimated temperature curve, can be seen in Fig. 14. The step temperature is controlled by the thermostat. The actual temperature, which reflects the accelerometer external ambient temperature, is gauged by a sensor named DS18B20. What illustrated in Fig. 14 is that the inside temperature lags outside temperature when the ambient temperature is dramatically changing. When the step of ambient temperature is $10^{\circ} \mathrm{C}$, the temperature difference between outside and inside can reach $4^{\circ} \mathrm{C}-6^{\circ} \mathrm{C}$. The lag time is up to $900 \mathrm{~s}$ when inner temperature reaches equilibrium. If the step of external environmental temperature is larger, the temperature difference between inside and outside will be bigger and the lag time will be longer.

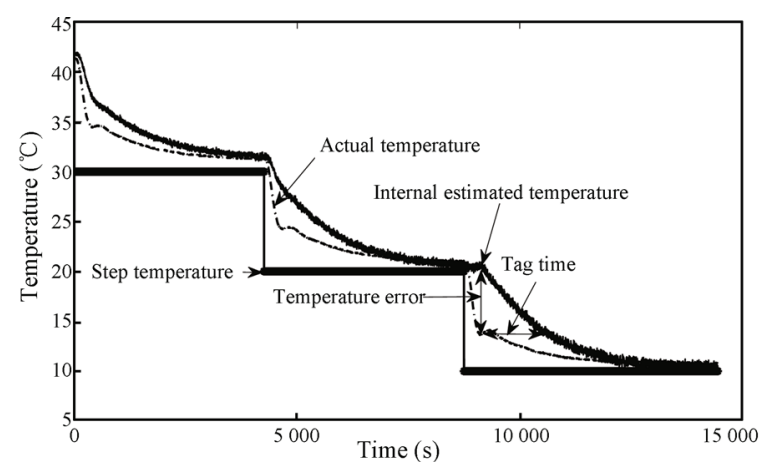

Fig. 14 Analysis for the inside and outside temperature

\subsection{Dynamic differential conduction model}

The internal components of accelerometer consist of metal coils and flexible pendulum modules. The thermal conductivity of these components is greater than that of air. The temperature of each component can be regarded as equal. The narrow space inside the accelerometer can be seen as an isothermal bulk. The main factor considered in temperature conduction is the process in which the heat from air passes through the metal case to the internal components ${ }^{[28,29]}$. The differential conduction model is illustrated as (10). The variable $\mu$ is the thermal conductivity of accelerometer.

$$
T_{i}(t)=T_{o}(t)+\sum_{j=0}^{n-1} \frac{(-1)^{i}}{\mu^{i}} \frac{\mathrm{d}^{i} T_{o}(t)}{\mathrm{d} t^{i}} .
$$

Taking the practical application and characteristics of acquisition circuit into account, the higher order differential terms in (10) can be ignored in order to simplify the large amount of calculation in the microcontroller. For example, ignoring the 5 th and higher differential terms, (10) is discretized as (11).

$$
\begin{aligned}
0= & L_{1} T_{o}\left(n-n_{1}\right)+L_{2} T_{o}\left(n-n_{2}\right)+L_{3} T_{o}\left(n-n_{3}\right)+ \\
& L_{4} T_{o}\left(n-n_{4}\right)+T_{o}(n)-T_{i}(n)
\end{aligned}
$$

where the variable $n_{i}(i=1,2,3,4)$ is the number of samples in the period of equal time. When the sampling frequency is high, it is essential to use the method of sampling interval so that to meet the third-order differential item requirement in (11). In the dynamic temperature test, the accelerometer output value and temperature inside thermostat can be collected in time. The internal temperature of accelerometer will be changed slowly. The slow process can be reflected by the slope of the output curve. Known from the static experiments, the internal temperature of accelerometer can be calculated by the output value. The size of output value reflects how high the internal temperature is, namely that the variable $T_{i}(n)$ in (11) can be estimated by the size of output value. The different rate of temperature change can be regulated by the thermostat so that each differential item in (11) can be known. Then, the coefficients $L_{1}, L_{2}, L_{3}$ and $L_{4}$ in (11) can be calculated. In the dynamic experiments, the sampling interval time is $90 \mathrm{~s}$. A data set of 8 elements are written down so that the temperature in $720 \mathrm{~s}$ can be remembered by an array. The difference and the rate of change of the temperature array will be known. The coefficients in the differential conduction model can be calculated. Then, the sampling interval time can be set as $90 \mathrm{~s}$, $120 \mathrm{~s}, 150 \mathrm{~s}$ and $180 \mathrm{~s}$. The coefficients in different sampling interval time can be calculated as shown in Table 4. Using these coefficients, the compensation value can be calculated by (11). The criterion to evaluate the compensation effect is given as the standard deviation. Adopting this criterion, the standard deviation of compensation result at different coefficients can be illustrated as Fig. 15.

Table 4 Coefficients for the differential model

\begin{tabular}{ccccc}
\hline Time (s) & $L_{1}$ & $L_{2}$ & $L_{3}$ & $L_{4}$ \\
\hline 60 & 0.25 & -2.07 & 3.07 & -6.36 \\
90 & -0.12 & -0.78 & 0.12 & -3.56 \\
120 & -0.24 & -0.54 & -0.55 & -2.44 \\
150 & -0.26 & -0.49 & -0.73 & -1.58 \\
180 & -0.3 & -0.52 & -0.66 & -1.12 \\
\hline
\end{tabular}

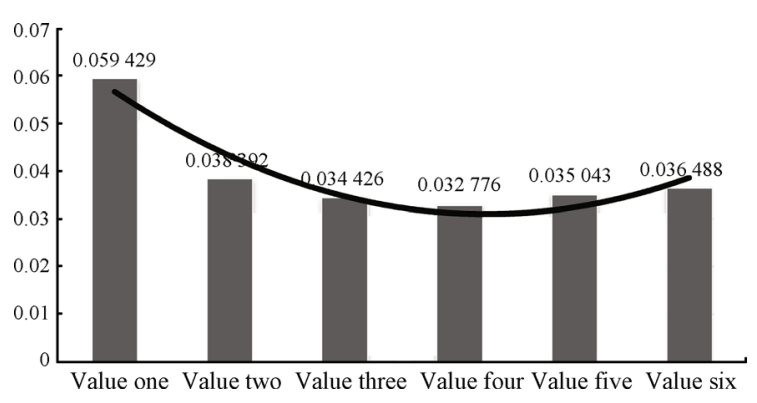

Fig. 15 Standard deviation at different interval time

The curve in Fig. 15 shows standard deviations in every condition. The value one in Fig. 15 is the volatility of output value compensated by the static model. The others 
in Fig. 15 are the volatility of output values compensated by the differential conduction model with the interval time $60 \mathrm{~s}, 90 \mathrm{~s}, 120 \mathrm{~s}, 150 \mathrm{~s}$ and $180 \mathrm{~s}$, respectively. From Fig. 15, it can be known that the static compensated error is the maximum value among the 6 items. That is also the reason why the static model is not applicable when the temperature changes rapidly and it is essential to study dynamic temperature conduction model. In the dynamic model, the standard deviation is different when the different coefficients are used. The optimal sampling interval time is $120 \mathrm{~s}$ as shown in Fig. 15. When the sampling interval time is $120 \mathrm{~s}$, the coefficients in Table 5 are adopted to compensate the accelerometer output value. The accelerometer internal temperature will be calculated as (12):

$$
\begin{aligned}
T_{i}(n)= & T_{o}(n)-0.24 T_{o}\left(n-n_{1}\right)-0.54 T_{o}\left(n-n_{2}\right)- \\
& 0.55 T_{o} 9\left(n-n_{3}\right)-2.24 T_{o}\left(n-n_{4}\right) .
\end{aligned}
$$

Table 5 Coefficients for the differential model

\begin{tabular}{cccccccc}
\hline Items & $L_{1}$ & $L_{2}$ & $L_{3}$ & $L_{4}$ & $L_{5}$ & $L_{6}$ & $L_{7}$ \\
\hline 3 & 0.14 & 0 & 0.86 & & & & \\
4 & 0.28 & 0 & 0 & 0.72 & & & \\
5 & 0.3 & 0 & 0.2 & 0 & 0.5 & & \\
6 & 0.31 & 0 & 0.15 & 0.13 & 0 & 0.41 & \\
7 & 0.3 & 0 & 0.19 & 0.03 & 0.15 & 0 & 0.33 \\
\hline
\end{tabular}

Substituting $T_{i}(n)$ in (12) by $T$ in (6) gives the output value compensation. When the external ambient temperature is changing as the curve of actual temperature shown in Fig. 14, the dynamic compensation is done and the result is shown in Fig. 16. From Fig. 16, it can be seen that the maximum error of output value without compensation is up to $1.38 \mathrm{mG}$. The maximum error of static compensation is $0.24 \mathrm{mG}$. The maximum error of dynamic differential compensation is $0.16 \mathrm{mG}$.

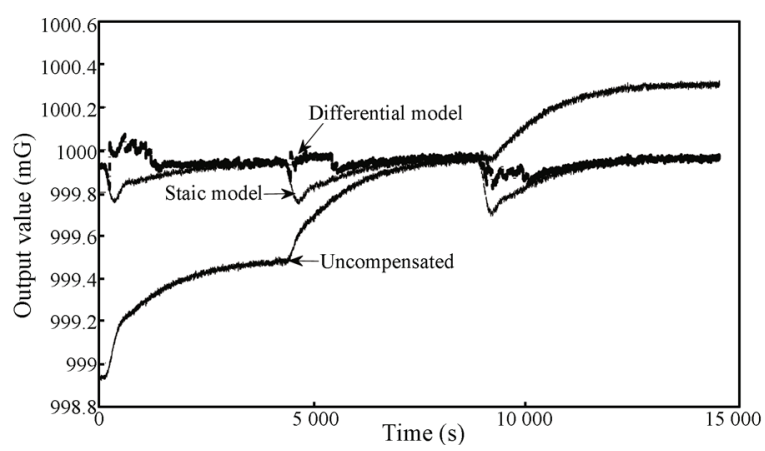

Fig. 16 Comparison between differential model and static model

\subsection{Dynamic weighted average model}

According to the analysis of the characteristics of temperature, the internal temperature of accelerometer can be regarded as the result of the combined effect of current ambient temperature and temperature in the past ${ }^{[30,31]}$. Therefore, the value of internal temperature can be considered as the weighted average of the outside temperature in differ- ent time. The weighted average model can be formulated as (13)

$$
\left\{\begin{array}{l}
T_{i}=L_{0} T_{0}\left(n_{0}\right)+L_{1} T_{0}\left(n_{1}\right)+\cdots+L_{k} T_{0}\left(n_{k}\right) \\
L_{0}+L_{1}+L_{2}+\cdots+L_{k}=1
\end{array}\right.
$$

where the variables $T_{o}\left(n_{0}\right), T_{o}\left(n_{1}\right), T_{o}\left(n_{2}\right), \cdots, T_{o}\left(n_{k}\right)$ respectively represent the external ambient temperature in the time $n_{0}, n_{1}, n_{2}, \cdots, n_{k}$. The variables $L_{0}, L_{1}, \cdots$, $L_{n}$ are the weighted coefficients in different time. The variable $k$ represents the number of compensation item in (13). In the dynamic experiments, the compensation item $k$ is changed from 3 to 7 . The sampling time interval is $120 \mathrm{~s}$ and 7 data points of equal time intervals are written down so that the temperature in $840 \mathrm{~s}$ can be remembered by an array. The 7 sample values at different time are selected for compensation. The weighted average coefficients of external ambient temperature in different time (past and current) can be calculated. Therefore, there is a group of 5 compensation coefficients because the compensation item number is 5. All the coefficients in (12) are shown in Table 5. Adopting the standard deviation criterion to evaluate the compensation effect, the standard deviation of compensation result at different coefficients can be illustrated as Fig. 17. The curve in Fig. 17 shows standard deviations in every condition. The value one in Fig. 17 is the volatility of output value compensated by the static model. The others in Fig. 17 are the volatility of output value compensated by the weighted average model with the compensation item 3 , $4,5,6,7$, respectively. From Fig. 17, it can be known that the static compensated error is maximum value among the 6 items. In the dynamic weighted average model, the standard deviation is decreasing with the increasing number of compensation item. In the application, the compensation items are selected as 7 items. The accelerometer internal temperature will be calculated as (14):

$$
\begin{aligned}
T_{i}(n)= & 0.3 T_{o}\left(n_{0}\right)+0.19 T_{o}\left(n_{2}\right)+0.03 T_{o}\left(n_{3}\right)+ \\
& 0.15 T_{o}\left(n_{4}\right)+0.33 T_{o}\left(n_{6}\right) .
\end{aligned}
$$

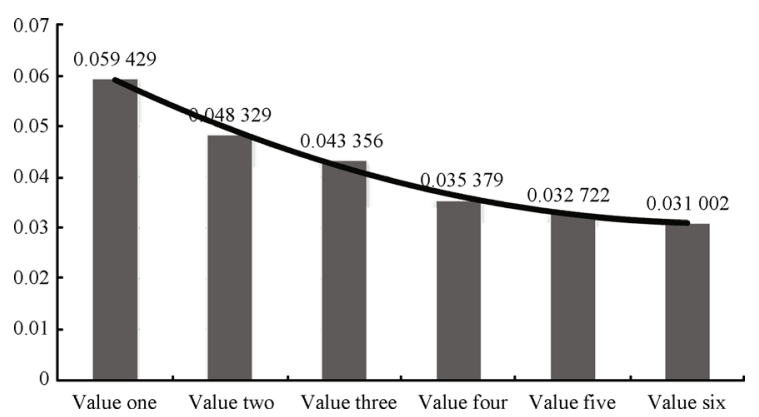

Fig. 17 Comparison between differential model and static model

Substituting $T_{i}(n)$ in (13) by $T$ in (6) gives the output value compensation. The weighted average compensation is done and the result is shown in Fig. 18. From Fig. 18, the maximum error of dynamic weighted average compensation is $0.12 \mathrm{mG}$. 


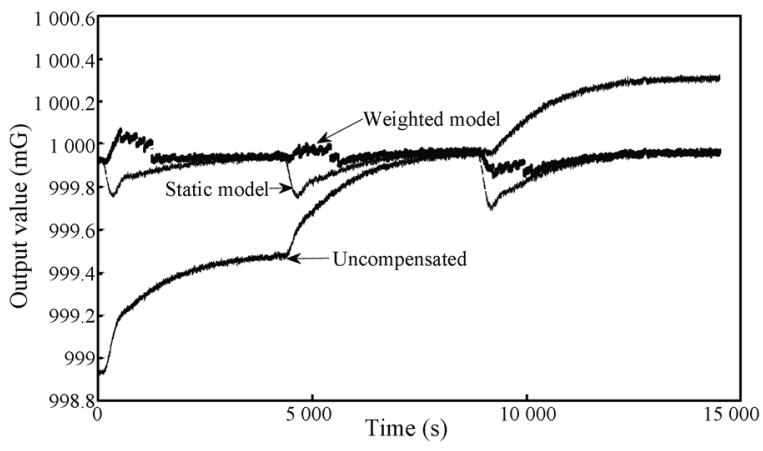

Fig. 18 Comparison between weighted average model and static model

\section{Conclusions}

This research focuses on the temperature characteristics of the accelerometer and temperature compensation. The method called orthogonal 12 points is adopted to find out the relationship between the output voltage and the temperature. Then, the compensation way is designed to calculate the difference voltage $\Delta E$. How to find out the coefficients of $\Delta E$ is a focus in the compensation program. Based on the compensation program, the output voltage after compensation is calculated as (8). Experiment data demonstrate that

1) The accuracy of output value is improved from $10^{-5} \mathrm{G}$ to $10^{-6} \mathrm{G}$.

2) The volatility of zero bias is improved from $9.8 \times$ $10^{-5} \mathrm{G}$ to $1.9 \times 10^{-6} \mathrm{G}$.

3) The volatility of scale factor is improved from $5.17 \times 10^{-4} \mathrm{~V} / \mathrm{G}$ to $1.31 \times 10^{-5} \mathrm{~V} / \mathrm{G}$.

Then, the dynamic conduction models are studied. Differential temperature conduction model can reflect the external ambient temperature rate. According to external temperature and temperature rate, the internal temperature can be estimated and output value can be compensated by the static model. Weighting average model reflects that the internal temperature can be regarded as result of the outside temperature effect at different time. The contradistinction of 3 dynamic compensation effect is shown in Table 6 . The advantage and disadvantage of 3 models can be seen in Table 6 . Both the simulation and experiment verified that 3 models can reduce the impact of temperature impose on accelerometers output value in dynamic process.

Table 6 Comparison between different models

\begin{tabular}{ccccc}
\hline Model & Error $(\mathrm{mg})$ & Deviation $(\mathrm{mg})$ & Resource (Byte) & Time (ms) \\
\hline A & 1.4057 & 0.3764 & 0 & 0 \\
B & 0.2919 & 0.0594 & 1152 & 4.3773 \\
C & 0.2721 & 0.0327 & 1568 & 4.9237 \\
D & 0.2243 & 0.0311 & 1376 & 4.4421 \\
\hline
\end{tabular}

Where the model A means that the output value is not compensated by any method. The model B means that the output value is compensated by the static method. The models $\mathrm{C}$ and $\mathrm{D}$ mean that the output values are compensated by differential conduct model and weighted average model, respectively. Shown in Table 6, the accuracy of accelerometer output value compensated by dynamic model is higher than that of static model. Compared with methods in [11-15], the differential conduction model and weighted average model are more favorable in practical application.

\section{References}

[1] K. Albert, T. David. High accelerometer, high performance solid state accelerometer development. Electronics Letters, vol. 38, no. 23, pp. 20-25, 1994.

[2] H. Hamacher. Microgravity characterisation and microgravity improvement for the columbus orbiting facility. In Proceedings of Space Satation Utilisation, ESOC, Darmstadt, Germang, SP-385, pp. 209-219, 1996

[3] R. V. Alauev, Y. V. Ivanov, D. M. Malyutin, V. Y. Raspopov, V. A. Dmitriev, S. P. Ermilov, G. A. Ermilova. High-precision algorithmic compensation of temperature instability of accelerometer's scaling factor. Automation and Remote Control, vol. 72, no. 4, pp. 853-860, 2011.

[4] R. Levy, Q. L. Traon, S. Masson, O. Ducloux, D. Janiaud, J. Guerard, V. Gaudineau, C. Chartier. An integrated resonator-based thermal compensation for vibrating beam accelerometers. In Proceedings of the IEEE Sensors, IEEE, Taipei, Taiwan, China, pp. 1-5, 2012.

[5] Y. J. Pan, L. L. Li, C. H. Ren, H. L. Luo. Study on the compensation for a quartz accelerometer based on a wavelet neural network. Measurement Science \& Technology, vol. 21, no. 10, pp. 102-115, 2010.

[6] Y. Yu, Y. S. Zhong. High precision two-stage robust temperature control for accelerometer unit. Acta Aeronautica et Astronautica Sinica, vol. 30, no. 6, pp. 1103-1108, 2009. (in Chinese)

[7] J. Lee, R. Jaewook. Temperature compensation method for the resonant frequency of a differential vibrating accelerometer using electrostatic stiffness control. Journal of Micromechanics and Microengineering, vol. 22, no. 9, pp. 172$184,2012$.

[8] S. Eichstat, A. Link, T. Bruns. On-line dynamic error compensation of accelerometers by uncertainty-optimal filtering. Measurement, vol. 43, no. 5, pp. 708-713, 2010.

[9] D. H. Li, X. Y. Gao, Z. X. Zhang, F. Zhou. Research on temperature property of piezoelectric vibration accelerometer based on cymbal transducer. Ferroelectrics, vol. 405 , no. 1, pp126-132, 2010.

[10] S. Shinomiya. Temperature error of two-phase induction generator-type accelerometer and its compensation. Electrical Engineering in Japan, vol. 96, no. 4, pp. 53-59, 1976. 
[11] L. T. Grigorie, R. M. Botez. The bias temperature dependence estimation and compensation for an accelerometer by use of the neuro-fuzzy techniques. Transactions of the Canadian Society for Mechanical Engineering, vol. 32, no. 3-4, pp. 383-340, 2008.

[12] H. Takao, Y. Matsumoto, M. Ishida, T. Nakamura, H. Tanaka, H. D. Seo. Three-dimensional vector accelerometer using SOI structure for high-temperature use. Electronics and Communications in Japan, vol. 79, no. 3, pp. 61-72, 1996.

[13] E. Gaura, R. Rider, N. Steele. Neural network based compensation of micromachined accelerometers for static and low frequency applications. In Proceedings of the 13th International Conference on Industrial and Engineering Applications of Artificial Intelligence and Expert Systems, New Orleans, USA, pp. 534-542, 2000.

[14] C. H. Ren, Y. J. Pan, J. F. Li, J. J. Liu. Two-dimensional compensation on time and temperature drift of quartzose flexible accelerometer based on neural network. Journal of Chinese Inertial Technology, vol. 15, no. 3, pp. 366-376, 2007 .

[15] L.T. Grigorie. The bias temperature dependence estimation and compensation for an accelerometer by the use of the neuro-fuzzy techniques. Transactions of the Canadian Society for Mechanical Engineering, vol. 32, no. 3-4, pp. 383-400, 2008 .

[16] Z. Xu, Y. F. Liu, J. X. Dong. Thermal bias drift compensation of MEMS accelerometer based on relevance vector machine. Journal of Beijing University of Aeronautics and Astronautics, vol. 39, no. 11, pp. 1558-1562, 2013.

[17] X. F. Li, D. H. Li, J. M. Gao, M. Pang. Temperature drift compensation algorithm based on BP and GA in quartz flexible accelerometer. Applied Mechanics and Mechanical Engineering, vol. 249-250, pp. 95-99, 2013.

[18] M. L. Ding, Q. D. Zhou, K. Song. A new method for accelerometer dynamic compensation based on CMAC. In Proceedings of the 4th International Symposium on Neural Networks: Advances in Neural Networks, Nanjing, China, pp. 667-675, 2007.

[19] L. Lang, J. G. Chen, M. P. Wu. Study of the temperature error model and the technology for compensating temperature of the quartz flexible accelerometer. Navigation and Control, vol. 8, no. 2,pp. 46-51, 2009. (in Chinese)

[20] R. V. Alaluev, Y. V. Ivanov, D. M. Malyutin, V. Y. Raspopov, V. A. Dmitriev, S. P. Ermilov, G. A. Ermilova. High-precision algorithmic compensation of temperature instability of accelerometer's scaling factor. Automation and Remote Control, vol. 72, no. 4, pp. 853-860, 2011.

[21] Test Methods for Single-axis Pendulous Servo Linear Accelerometers, National Science and Technology Commission, GJB 1037-2004, 2004. (in Chinese)
[22] Verification Equipment of Linear Accelerometer by Earth's Gravitation. State Administration of Quality Supervision and Inspection Quarantine, GJB 1071-2011, 2011. (in Chinese)

[23] V. M. Gotlib, E. N. Evlanov, B. V. Zhbkov,V. M. Linkin, A. B. Manukin, S. N. Podkolzin, V. I. Rebrov. High-sensitivity quartz accelerometer for measurements of small accelerations of spacecraft. Cosmic Research, vol. 42, no. 1, pp. 54$59,2004$.

[24] F. Li, L. Y. Wang, J. H. Zhao. Research on error compensation for oil drilling angle based on ANFIS. In Proceedings of the 3th International Conference on Intelligent Computing, Springer, Qingdao, China, pp. 730-737, 2007.

[25] B. V. Amini, F. Ayazi. Micro-gravity capacitive silicon-oninsulator accelerometers. Journal of Micromechanics and Microengineering, vol. 15, no. 11, pp. 2113-2120, 2005.

[26] M. A. Barulina, V. E. Dzhashitov, V. M. Pankratov, M. A. Kalinin, A. A. Papko. Mathematical model of a micromechanical accelerometer with temperature influences, dynamic effects, and the thermoelastic stress-strain state taken into account. Gyroscopy and Navigation, vol. 1, no. 1, pp. 52-61, 2010.

[27] K. I. Lee, H. Takao, K. Sawada, H. D. Seo M. Ishida. Improvement of thermal response in temperature controlled precise three-axis accelerometer with stabilized characteristics over a wide temperature range. In Proceedings of the 13th International Conference on Solid-State Sensors, Actuators, and Microsystems, IEEE, Seoul, South Korea, vol. 1, pp. 800-803, 2005.

[28] J. Mackley, S. Nahavandi. Active temperature compensation for an accelerometer based angle measuring device. In Proceedings of the Intelligent Automation and Control Trends, IEEE, Seville, Spain, pp. 383-388, 2004.

[29] A. Stefani, W. Yuan, C. Markos, H. K. Rasmussen, S. Andresen, R. Guastavino, F. K. Nielsen, B. Rose, O. Jespersen, N. Herholdt-Rasmussen, O. Bang. Temperature compensated, humidity insensitive, high-Tg TOPAS FBGs for accelerometers and microphones. In Proceedings of the 22nd International Conference on Optical Fiber Sensors, Beijing, China, vol. 8421, pp. 100-115, 2012.

[30] X. T. Yu, L. Zhang, L. R. Guo, F. Zhou. Identification for temperature model of accelerometer based on proximal SVR and particle swarm optimization algorithms. Journal of Control Theory and Applications, vol. 10, no. 3, pp. 349353, 2012.

[31] J. Lundberg, A. Parida, P. Söderholm. Running temperature and mechanical stability of grease as maintenance parameters of railway bearings. International Journal of Automation \& Computing, vol. 7, no. 2, pp.160-166, 2010. 


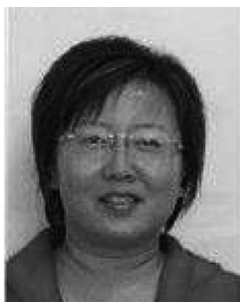

Jing-Min Gao received the B. Sc. and M. Sc. degrees in hydraulic and pneumatic technology from Inner Mongolia University, China in 1995. She received the Ph. D. degree from Beijing Institute of Technology, China in 1999. Now, she is a professor in Beijing Information Science \& Technology University.

Her research interests include intelligent detection technology and information fusion and process.

E-mail: gaojm_biti@163.com

ORCID iD: 0000-0002-3555-2927

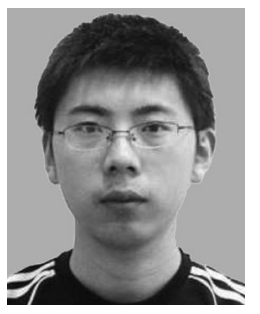

Ke-Bei Zhang received the B. Sc. degree in automation from Beijing Information Science \& Technology University, China in 2010. Now, he is a Ph. D. candidate in Beijing Institute of Control Engineering, China.

His research interests include automation instrumentation and rigid body attitude determination.

E-mail: zhangkb.2008@163.com (Corresponding author)
ORCID iD: 000-0003-4418-5134

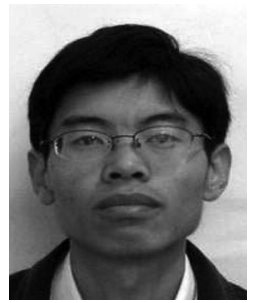

Fu-Bin Chen received the B. Sc. degree in signal and information processing from Harbin Institute of Technology, China, in 2002. He is currently a lecturer at Beijing Information Science \& Technology University, China.

His research interests include circuit design, intelligent detection technology and data fusion.

E-mail: chenfubin@bistu.edu.cn

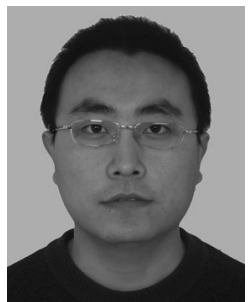

Hong-Bo Yang received the Ph. D. degree in detection and information fusion from the Chinese Academy of Science in 2005. He is currently an associate professor at Beijing Information Science \& Technology University.

His research interests include signal and information processing and intelligent information processing.

E-mail: anonbo@163.com 